\title{
$\beta$-Conglycinin Regulates Intestinal Immunity through the CIITA-mediated MHC II-PI3K/Akt/mTOR Pathway in Hybrid Grouper: The Ameliorative Effects of Sodium Butyrate (NaB)
}

Bin Yin

Guangdong Ocean University

Hongyu Liu

Guangdong Ocean University

Beiping Tan ( $\sim$ bptan@126.com )

Guangdong Ocean University

Xiaohui Dong

Guangdong Ocean University

Shuyan Chi

Guangdong Ocean University

Qihui Yang

Guangdong Ocean University

Shuang Zhang

Guangdong Ocean University

\section{Research Article}

Keywords: hybrid grouper (Epinephelus fuscoguttatus $\nabla \times E$. lanceolatus $\varangle$ ), $\beta$-conglycinin, sodium butyrate, serum immunity, intestinal histopathology, intestinal inflammation, MHC II PI3K/Akt/mTOR

Posted Date: January 27th, 2021

DOI: https://doi.org/10.21203/rs.3.rs-148926/v1

License: (c) (1) This work is licensed under a Creative Commons Attribution 4.0 International License. Read Full License 


\section{Abstract}

We investigated the effects of low and high doses of $7 \mathrm{~S}$ and the ameliorative effects of $\mathrm{NaB}$ (based on high-dose 7S) on the growth performance, serum immunity, distal histopathology, and CIITA-mediated $\mathrm{MHC}$ II-PI3K/Akt/mTOR pathway in hybrid groupers. The results revealed that the specific growth rate of groupers significantly increased, decreased, and increased in the low-level 7S (bL), high-level 7S (bH) high-level 7S plus $\mathrm{NaB}$ (bH-NaB) groups, respectively. The feed coefficient ratio was significantly increased in the $\mathrm{bH}$ and bH-NaB groups, whereas serum levels of IFN- $\mathrm{y}$, IL-1 $\beta$, and TNF-a were upregulated in the bH group. The intestinal diameter/plica height ratio was significantly increased in the bH group. Furthermore, there were increases in nitric oxide, nitric oxide synthase (NOS), and peroxynitrite anion $\left(\mathrm{ONOO}^{-}\right)$in the bH group, and decreases in $\mathrm{NOS}^{2}$ and $\mathrm{ONOO}^{-}$in the bH-NaB group. The mRNA levels in distal intestine of TSC1, mTOR C2, CIITA, and CREB1 were significantly upregulated in all three treatment groups, whereas those of IKKa, Rheb, mTOR C1, mLST8, EIF4B, NFY, GILT, and AEP were upregulated and downregulated in the bH and bH-NaB groups, respectively. These indicate 7S has a regulatory effect on serum immunity and affect distal intestinal development by modulating hindgut injury-related parameters. Within the distal intestinal tract, 7S can induce intestinal inflammation by activating the CIITA-mediated MHC II-PI3K/Akt/mTOR pathway, which eventually manifests as a reduction in growth performance. Supplementing feed with $\mathrm{NaB}$ represents an effective approach for enhancing serum immunity, and also protects the intestines from damage caused by high-dose $7 \mathrm{~S}$.

\section{Introduction}

The hybrid grouper (Epinephelus fuscoguttatus $\square \times$ E. lanceolatus $\bigotimes$ ) is characterized by a rapid growth rate and desirable meat quality, which is of high economic value. As a typical carnivorous marine fish, hybrid grouper typically require up to $50-55 \%$ protein in their feed ${ }^{1}$, which is generally provided by fishmeal containing high levels of nitrogen and phosphorus. In addition, soybean meal is also a widely available and inexpensive source of protein for hybrid grouper diets. However, hybrid grouper have a low tolerance to soybean meal, which can make up approximately $40-60 \%$ of fishmeal diets ${ }^{2}$. Although a high intake of soybean meal can lead to intestinal inflammation in hybrid groupers ${ }^{3}$, the prevision of appropriate amounts of this meal can enhance immunity to a certain extent, thereby improving growth performance. These effects are widely believed to be attributable to the immunogenicity of the soy glycinin (11S) and $\beta$-conglycinin (7S) contents of soybean meal ${ }^{4}$, which are the main components of soybean storage proteins. $\beta$-conglycinin has a molecular weight of 140-180 kD, and accounts for approximately $30 \%$ of the soy protein content. Most of the $7 \mathrm{~S}$ ingested as a nutrient is absorbed by the intestinal tract, and contributes little to the antigenic effect. In piglets, 7S allergic reactions mainly induce a reduction in the height of the intestinal villi and thereby reduces the efficiency of nutrient absorption ${ }^{5}$, whereas in grass carp, 7S can promote an increase in reactive oxygen species (ROS) production in the distal intestine, leading to disruption of the structural integrity of epithelial cells ${ }^{6}$. However, the mechanisms underlying the intestinal tolerance to $7 \mathrm{~S}$ and the intestinal inflammation caused by this compound in hybrid grouper have yet to be determined. 
Short-chain fatty acids include acetic acid, propionic acid, and butyric acid, with the latter playing a major role in regulating the organism metabolism ${ }^{7}$. It has been established that the hydrolysis of sodium butyrate ( $\mathrm{NaB}$ : the sodium salt form of butyric acid) to butyric acid has a wide range of physiological effects, providing an energy source for intestinal cells, promoting intestinal cell differentiation and proliferation, and maintaining intestinal morphology to facilitate digestion and absorption ${ }^{8}$. Accordingly, given its unique nutritional and physiological functions and lack of polluting and harmful residues, $\mathrm{NaB}$ has become a valuable additive that is used to supplement the diets of cultured aquatic animals.

The intestinal tract of fish is well supplied by nerves and microvasculature, and is often referred to as the second brain of the fish. Moreover, it also contributes to the provision of $70 \%$ of fish immunity.

Accordingly, as the most fully functional immune organ in fish, the intestines play a dual role in providing tolerance to feed antigens and immune defense. Unlike mammals, fish epitopes are primarily distributed in the hindgut, and consequently, compared with the foregut and midgut, the hindgut is more susceptible to attack by antigens present in feed, which can potentially cause intestinal inflammation. In this regard, studies have shown that the major histocompatibility complex MHC II plays an important role in identifying and presenting foreign antigens, and research in our laboratory has revealed that intestinal inflammation in hybrid grouper caused by ingesting feed containing high levels of soybean meal is related to an abnormal activation of the PI3K/Akt/mTOR signaling pathway. However, it has yet to be established whether this activation is due to the recognition of foreign antigens by MHC II. Furthermore, it has also been found that $\mathrm{NaB}$ can significantly inhibit the Akt/mTOR signaling pathway, and thus we speculate that by inhibiting PI3K/Akt/mTOR, NaB could be used to relieve the intestinal inflammation caused by $7 \mathrm{~S}$.

In this study, we therefore used hybrid groupers as experimental animals to evaluate the hypothesis that dietary $\beta$-conglycinin might affect growth by regulating serum immunity, distal intestinal histopathology, epithelial injury, and the PI3K/Akt/mTOR signaling pathway. In addition, we also investigated the potential protective effects of appropriate doses of $\mathrm{NaB}$ against high levels of dietary $7 \mathrm{~S}$. We anticipate that the findings of this study will provide a reference for research on improving the tolerance of hybrid grouper to soybean meal and reveal the mechanisms underlying the protective effects of $\mathrm{NaB}$ on the distal intestine.

\section{Methods}

The animal protocol used in the present study was approved by the ethics review board of Guangdong Ocean University, and all experimental procedures concerning animals were performed in accordance with the National Centre for the Replacement Refinement \& Reduction of Animals in Research (The ARRIVE guidelines 2.0) and relevant Chinese policies.

\section{Feeding trial and challenge test}


The juvenile hybrid grouper used in this study were purchased from a commercial hatchery on East Island (Zhanjiang, China), and were derived from the same batch of artificially hatched fish. Prior to experimentation, the fish were acclimated to the local environment by placing in an outdoor pond of the Biological Research Base of Guangdong Ocean University for 1 week, during which time they were provisioned with commercial feed.

Having acclimated, 480 healthy uniformly sized hybrid groupers were selected and randomly divided into four groups, each with four replicates of $30 \mathrm{fish}$, and the fish were cultured in 300-L glass fiber buckets. During the culture period, the fish were fed twice daily (at 08:00 and 16:00) to apparent satiety with a water change of approximately $70 \%$. Dissolved oxygen levels in the containers were maintained at $\geq 7.00$ $\mathrm{mg} / \mathrm{L}$ via continuous aeration using air stones, whereas water temperature, $\mathrm{pH}$, and ammonia nitrogen content were maintained at $29.00 \pm 1.30^{\circ} \mathrm{C}, 7.8-8.1$, and $<0.09 \mathrm{mg} / \mathrm{L}$, respectively. At the end of the 8week culture period, 40 fish per treatment were randomly selected for a challenge test (10 fish per replicate). Each fish was injected intraperitoneally with $200 \mu \mathrm{L}$ of Vibrio parahaemolyticus at a concentration of $7.41 \times 10^{8} \mathrm{CFU} / \mathrm{ml}$, and thereafter the fish were continuously observed for 1 week, with mortality being recorded daily.

\section{Diet formulations}

Experimental fish were provided with one four diets containing protein and fat at levels of approximately $48 \%$ and $10 \%$, respectively, which were prepared using redfish meal, casein, and gelatin as the main protein sources, and fish oil and soy lecithin as the main lipid sources. As treatments, the diet was supplanted as follows: $0 \% 7 \mathrm{~S}, 0 \% \mathrm{NaB}$ (fishmeal group, FM); $1.50 \%$ 7S (low-level 7S, bL), $0 \% \mathrm{NaB} ; 6.00 \%$ $7 \mathrm{~S}, 0 \% \mathrm{NaB}$ (high-level 7S, bH); $6.00 \%$ 7S, $0.13 \% \mathrm{NaB}$ (high-level 7S with NaB, bH-NaB) (Table S1). The three experimental diets were also supplemented with methionine and lysine to same level as that in the FM diet. The essential amino acid profiles of the diets used in this experiment are shown in Table S2. Purified 7S was purchased from the China Agricultural University (Patent No. 200410029589.4, China). For use in the experimental diets, water was added to the purified $\beta$-conglycinin several times in small amounts, stirred thoroughly, and the $\mathrm{pH}$ of the solution was adjusted to 7.2. The 7S solution was then placed in a glass petri dish and dried for $72 \mathrm{~h}$ in a low-temperature freeze dryer. After crushing the different raw materials of the diet through 380- $\mu \mathrm{m}$ meshes, the components mixed in a stepwise manner, adding fish oil, soybean lecithin, and $30 \%$ water and mixing evenly. The resulting mixture was then placed into a feed pelletizer, which produced feed pellets of $2.5 \mathrm{~mm}$ in diameter. The pellets were air-dried at room temperature until the moisture content was approximately $10 \%$ (approx. $48 \mathrm{~h}$ ), sealed in a selfsealing bag, and stored at $-20^{\circ} \mathrm{C}$ until required. The $30 \%$ microencapsulated $\mathrm{NaB}$ used in this study was kindly provided by Shanghai Menon Animal Nutrition Technology Co., Ltd.

\section{Sample collection}


After the end of the 8-week breeding experiment, the fish in each bucket were fasted for $24 \mathrm{~h}$ and then counted and weighed. For each replicate, two fish were randomly selected, from which blood was drawn using a $1 \mathrm{~mL}$ syringe. After being left to stand for $12 \mathrm{~h}$, the blood samples were centrifuged for $10 \mathrm{~min}$ at $5000 \mathrm{~g} / \mathrm{min}$ and $4^{\circ} \mathrm{C}$. The serum thus obtained was collected and stored at $-80^{\circ} \mathrm{C}$ for the determination of serum immune indicators. A further six fish were randomly selected for each replicate, from which the intestines were gently removed, and the segment of intestine near the cloacal hole was cut as the distal intestine (DI). Two of the DI segments for each replicate were placed in $4 \%$ formaldehyde solution for the preparation of Alcian Blue-Periodic acid-Shiff (AB-PAS) stained intestinal sections, and a further two DI segments were placed in liquid nitrogen for the determination of hindgut-related enzyme activities. After the DI tissue had been thawed on ice, dried with qualitative filter paper, and weighed, a sample was homogenized in homogenization medium ( $0.8 \%$ sodium chloride solution) in an ice bath at a sample: medium ratio of $1: 9$ (mass to volume ratio), centrifuged at $5000 \mathrm{~g} / \mathrm{min}$ for $10 \mathrm{~min}$ at $4^{\circ} \mathrm{C}$, and the supernatant was collected for analysis. The remaining two DI samples from each replicate were placed in RNA latter, and after being left to stand for $12 \mathrm{~h}$, were stored in a freezer at $-80^{\circ} \mathrm{C}$ for subsequent determination of related gene expression.

\section{Determination of growth and DI evaluation indicators}

Parameters of interest were determined as follows:

WGR $($ weight gain rate, $\%)=100 \times($ final weight - initial weight $) /$ initial weight

SGR $($ specific growth rate, $\%)=100 \times[\ln ($ final weight $)-\ln$ (initial weight) $] /$ days of experiment

SR $($ survival rate, $\%)=100 \times$ final fish number/initial fish number

FCR (feed coefficient ratio) $=$ dry feed consumed $/$ weight gain

$\mathrm{Id} / \mathrm{Ph}=$ intestinal diameter/plica height

\section{Immune index determinations}

Serum immune and DI injury indices were determined using Shanghai Enzyme-Linked ELISA immunoassay kits (Full-wavelength microplate reader-1510). NO was tested by Nitric Oxide assay kit from Nanjing Jiancheng Bioengineering Institute. AB-PAS sections of DI were prepared by Wuhan Servicebio Biological Technology Co., Ltd. Observations were made using a Leica DM 6000 optical microscope, and measurements and photographs were taken using cellSens Standard 1.8 and LAS 3.8 software, respectively, with 10 randomly selected plicae and muscle layers being measured in each section. 
Total RNA from hybrid grouper DI was extracted using a TranZol Up Plus RNA kit (Beijing TransGen Biotechnology Co., Ltd) in accordance with the manufacturer's instructions. The integrity of the extracted RNA was assessed by $1 \%$ agarose gel electrophoresis, and a NanoDrop ND-2000 spectrophotometer (Thermo Scientific, USA) was used to determine the purity and concentration of RNA. RNA samples of sufficient quality should run as a clear and complete band, and have an $\mathrm{OD}_{260} / \mathrm{OD}_{280}$ ratio of between 1.8 and 2.0. A PrimerScript ${ }^{\mathrm{TM}}$ RT-PCR Kit (TaKaRa, Kusatsu, Japan) was used to reverse transcribe total RNA to CDNA, which was stored at $-20^{\circ} \mathrm{C}$ prior to RT-PCR analysis. Specific primers for RT-PCR (Table S3) were designed using Primer Premier 5.0 software based on full-length transcriptome sequences of hybrid grouper in the NCBI Sequence Read Archive with accession number PRJNA664416. All the real-time PCR reactions were run in an Applied Biosystems 7500 Real-Time PCR System (Life Technologies, Carlsbad, CA, USA) using a SYBR $®$ Premix Ex Taq ${ }^{\text {TM }}$ Kit (Takara). The $\beta$-actin gene was used as an internal reference to normalize the amplification efficiency of each target gene, and the mRNA expression levels of genes were compared and analyzed using $2^{-\Delta \Delta C T}$ methods according to Livak et al. ${ }^{9}$.

The protein supernatant was extracted from intestinal tissues. Protein concentration was determined using the BCA method, supplemented with PBS and loading buffer to adjust the final protein concentration of the sample to $2 \mathrm{ug} / \mathrm{uL}$, and boiled in boiling water for 10 minutes. and finally quickly put the sample on ice, moved to $-80^{\circ} \mathrm{C}$ refrigerator until used. SDS-PAGE was used to separate the protein samples (10 uL sample per hole). The steps of electrophoresis, membrane transfer, closure and antibody incubation are performed according to the previous methods in our lab ${ }^{10}$. The following antibodies were used in this study: antibodies against PI3 Kinase p85 (4292), phospho-PI3 Kinase (Tyr458, 4228S), Akt (9272S), phospho-Akt (Ser473, 9271S) and GAPDH (2118S) were all purchased from Cell Signaling Technology. On account of mammalian antibodies being used, amino acid sequences of studied protein from hybrid grouper were aligned in the NCBI database (https://blast.ncbi.nlm.nih.gov/Blast.cgi) to check the identity of the antibodies. The western bands were quantified using NIH Image 1.63 software.

\section{Statistical analyses}

All data were subjected to one-way analysis of variance followed by Tukey multiple range tests to determine significant differences among treatment groups using SPSS v. 22 (IBM, USA) at a significance level of $P<0.05$, as described by Guo et al. ${ }^{11}$. The results are presented as the means $\pm \mathrm{SE}$.

\section{Results}

\section{Growth performance and challenge test}

The results obtained for the growth performance of fish in different groups are presented in Table 1. These results indicated that compared with the FM group, the final body weight (FBW), weight gain rate (WGR), and specific growth rate (SGR) were all significantly higher in the bL group and lower in the bH group, whereas no significant differences were observed between the FM and bH-NaB groups. The feed 
coefficient ratio (FCR) in the bH group was found to differ significantly from that in the other three groups, although there was no significant difference between the FM and bL groups. In contrast, we detected no significant differences among the four groups with respect to survival rate (SR).

The cumulative mortality of fish in response to injecting with $V$. parahaemolyticus is shown in Figure S1, which indicates that fish in the $\mathrm{bH}$ and bH-NaB groups suffered heavier mortalities than those in the FM and bL groups, with all groups reaching an asymptote at approximately 5 days post-injection. Among the different groups, the bH group showed a sustained and rapid increase in cumulative mortality from the first day post-injection, whereas in the bH group, mortality increased slowly over the first 3 days after injection, although thereafter showed a rapid increase.

Table 1

Growth parameters and feed utilization of juvenile hybrid grouper (Epinephelus fuscoguttatus $\square$ E. lanceolatus $\nabla$ ) fed the experimental diets for 8 weeks.

\begin{tabular}{|c|c|c|c|c|}
\hline & FM & bL & bH & bH-NaB \\
\hline IBW (g) & $7.70 \pm 0.05$ & $7.72 \pm 0.06$ & $7.70 \pm 0.04$ & $7.69 \pm 0.05$ \\
\hline FBW (g) & $50.60 \pm 0.26^{b}$ & $56.21 \pm 2.71^{c}$ & $42.09 \pm 6.52^{\mathrm{a}}$ & $51.11 \pm 1.31^{\mathrm{b}}$ \\
\hline WGR (\%) & $557.22 \pm 3.33^{b}$ & $636.50 \pm 11.37^{c}$ & $464.18 \pm 6.76^{a}$ & $608.87 \pm 32.51^{b c}$ \\
\hline SGR (\%/day) & $3.36 \pm 0.01^{\mathrm{b}}$ & $3.57 \pm 0.03^{c}$ & $3.09 \pm 0.02^{\mathrm{a}}$ & $3.49 \pm 0.16^{b c}$ \\
\hline SR (\%) & $100.00 \pm 0.00$ & $100.00 \pm 0.00$ & $99.17 \pm 0.83$ & $100.00 \pm 0.00$ \\
\hline FCR & $0.81 \pm 0.05^{\mathrm{a}}$ & $0.77 \pm 0.05^{\mathrm{a}}$ & $1.52 \pm 0.21^{c}$ & $1.04 \pm 0.08^{b}$ \\
\hline \multicolumn{5}{|c|}{$\begin{array}{l}\text { Value show means } \pm \text { SE }(n=4) \text {; Significance was evaluated by one-way ANOVA followed by Tukey's } \\
\text { multiple range tests. FM, control diet; bL, containing } 1.5 \% 7 \mathrm{~S} \text { diet, bH, containing } 6 \% \text {, } 7 \mathrm{~S} \text { diet, bH-NaB, } \\
\text { containing } 6 \% 7 S \text { and } 0.13 \% \text { SB diet. IBW, initial body weight; FBW, final body weight; WGR, weight } \\
\text { gain rate; SGR, specific growth rate; SGR, survival rate; FCR, feed coefficient ratio. a, b, c, dMean values } \\
\text { among all treatments with different letters were significantly different when the interaction was } \\
\text { significant }(P<0.05) \text {. }\end{array}$} \\
\hline
\end{tabular}

\section{Serum immune indices}

The serum immune indices determined for the different groups are presented in Figure. 1. Compared with the FM group, we detected significant increases in serum IFN- $y$ in the $\mathrm{bL}, \mathrm{bH}$, and bH-NaB groups, among which the bH-NaB group showed the most significant difference. The levels of IL-1 $\beta$ were found to be significantly increased in the bH group and significantly reduced in the $\mathrm{bL}$ and $\mathrm{bH}-\mathrm{NaB}$ groups, although no significant differences were detected between the bL and bH-NaB groups. With respect to TNF-a, we observed significantly downregulated and upregulated levels in the bL and bH groups, respectively, compared with the FM group, whereas in contrast, there was no significant difference between the bH$\mathrm{NaB}$ and FM groups. 


\section{Distal intestinal histopathological evaluation and damage indicators}

The results obtained for the different groups with respect to distal intestinal histopathology evaluated by AB-PAS staining, developmental status assessment, and damage indicators determined are presented in Figures 2, 3, and 4, respectively. As shown in Figure 3, the intestinal diameter/plica height ratios (Id/Ph) obtained for the $\mathrm{bL}$ and $\mathrm{bH}-\mathrm{NaB}$ groups were significantly higher than that for the FM group, and significantly lower than that for the bH group. Among the four groups, the bH group was found to have the lowest density of mucus cells, whereas we detected no significant differences among the other three groups.

Compared with the FM group, the levels of nitric oxide (NO) were significantly upregulated in the bH and bH-NaB groups (Figure 4). Similarly, although the levels of NO in the bL groups were somewhat lower, they were still significantly higher than those in the FM group. Whereas we observed that levels of nitric oxide synthase (NOS) in the bH group were significantly higher than those in the $\mathrm{FM}, \mathrm{bL}$, and bH-NaB groups, we detected no significant difference among the FM, bL, and bH-NaB groups. Moreover, levels of the peroxynitrite anion ( $\left.\mathrm{ONOO}^{-}\right)$were found to be lowest in the bL group, whereas no significant differences were observed among the $\mathrm{FM}, \mathrm{bH}$, and bH-NaB groups.

\section{Distal intestinal immune-related gene expression and protein expression}

The effects of $7 \mathrm{~S}$ and $\mathrm{NaB}$ on the distal intestine of hybrid grouper are presented in Figure 5. Compared with the FM group, the mRNA levels of PI3K, RS5, PRAS40, p70 S6K, and PRR5 were upregulated in the bL and bH groups, and were significantly upregulated in the bH-NaB group. Similarly, we observed that the mRNA levels of 3-PDK1, mTOR C1, TEL2, EIF4E, EIF4B, Sin1, NFY, MHC I, GILT, AEP, and TCR were all upregulated in the $\mathrm{bL}$ and $\mathrm{bH}-\mathrm{NaB}$ groups, and were significantly upregulated in the bH group. Although Rheb, Raptor, mLST8, and 4EBP1 mRNA levels were also significantly upregulated in the bH group, no changes were detected between the $\mathrm{bL}$ and $\mathrm{bH}-\mathrm{NaB}$ groups and the FM group.

In addition, the bL, bH and bH-NaB groups all showed significantly higher levels of TSC1, mTOR C2, 4EBP1, CIITA and CREB1 mRNA compared with the FM group, whereas no significant differences were detected between the $\mathrm{bH}$ and $\mathrm{bH}-\mathrm{NaB}$ groups. Although we found that the mRNA levels of deptor, RICTOR, SGK1, RFX5, and MHC II in the bH and bH-NaB group were significantly higher than those in the FM and bL groups, no significant differences were observed between the FM and bL groups or between the bH and bH-NaB groups. PKC mRNA levels were upregulated in the bH-NaB group and downregulated in the $\mathrm{bL}$ group, and those of RhoA were upregulated in the $\mathrm{bL}$ and $\mathrm{bH}-\mathrm{NaB}$ groups compared with the FM group. Moreover, the mRNA expression of CD4 was significantly downregulated in the bL, bH, and bH-NaB groups, whereas we detected no significant differences among the four groups with respect to TSC2 and S6. 
For western blot analysis of PI3K-Akt-mTOR (Figure 6), the expressions of P-PI3K/GAPDH and PAkt/GAPDH and P-Akt/mTOR were significantly up-regulated in bL group, meanwhile, the PI3K/GAPDH, P$\mathrm{PI3K} / \mathrm{GAPDH}$ and P-PI3K/PI3K were significantly up-regulated in bH group. Compared with bH group, the $\mathrm{PI3K} / \mathrm{GAPDH}, \mathrm{Akt} / \mathrm{GAPDH}$ and P-PI3K/PI3K were all significantly up-regulated after supplementing $\mathrm{NaB}$ in bH-NaB group.

\section{Discussion}

In general, due to the incomplete development of the immune system in juvenile animals, the intestines of individuals often show an allergic reaction to 7S, resulting in metabolic disorders and adverse physiological and biochemical reactions, which can have the effect of disrupting the digestion and absorption of nutrients in the intestines ${ }^{12}$. Interestingly, a low dose of $7 \mathrm{~S}$ can often induce a response similar to that obtained with immunostimulants ${ }^{13}$, which is often manifested in terms of modified growth performance. For example, in turbot (Scophthalmus maximus L.), a reduction in feed utilization efficiency following ingestion of feeds containing 4-8\% 7S has been observed to result in a significant reduction in specific growth rate (SGR) ${ }^{14}$. These findings were similar to those obtained in the present study, in which we observed that hybrid grouper receiving a diet containing $8 \% 7 \mathrm{~S}$ showed a significant reduction in SGR and weight gain (WG). However, whereas in turbot, the effect of ingesting of a diet containing $2 \% 7 \mathrm{~S}$ did not differ significantly from that of a control diet with respect to SGR, we observed an increase in SGR in hybrid grouper fed a diet supplemented with low-dose 7S. Moreover, in turbot, it was found that the level of soybean supplantation and WG showed a first-degree relationship ${ }^{15}$. Hybrid groupers can, however, tolerate up to $30-50 \%$ replacement of fishmeal with soybean meal in their feed, and experience no adverse effects on growth, even at soybean meal substitution levels of $10 \%$ and $30 \%$, WG increased while did not show a significant difference ${ }^{16}$. These observations thus indicate that hybrid grouper may be more tolerant to soybean meal than are turbot, and therefore we speculate that for turbot, the addition of $2 \%$ soybean globulin is higher than the dose used for immune enhancement.

In the present study, we found that the addition of sodium butyrate $(\mathrm{NaB})$ significantly increased SGR in the bH-NaB group compared with that in the bH group, indicating that $\mathrm{NaB}$ can be effective in ameliorating the growth inhibition effects attributable to high doses of $7 \mathrm{~S}$. We also found that the feed coefficient ratio of fish in the $\mathrm{bH}$ and bH-NaB groups was significantly higher than that in the $\mathrm{FM}$ and bL groups, whereas the addition of $\mathrm{NaB}$ significantly reduced the FCR, indicating that $\mathrm{NaB}$ may enhance the growth performance by improving the feed utilization of hybrid grouper, which is similar to results obtained for grass carp ${ }^{8,17}$. We speculate that this effect of $\mathrm{NaB}$ is associated with an increase intestinal antioxidant capacity and enhancement of intestinal immunity.

To further investigate the effects of $7 \mathrm{~S}$ and $\mathrm{NaB}$ on the disease resistance of the hybrid grouper, we conducted a 7-day challenge test using Vibrio parahaemolyticus injected into fish at a concentration of $7.41 \times 10^{8} \mathrm{CFU} / \mathrm{mL}$ at the end of the breeding experiments. We accordingly observed the highest cumulative mortality in the bH group, thereby indicating that high doses of $7 \mathrm{~S}$ can significantly reduce 
disease resistance in hybrid groupers, whereas resistance was enhanced to some extent in groupers fed a diet supplemented with $\mathrm{NaB}$. Interestingly, whereas the trend in mortality in the bH group showed a consistent and rapid increase during the first 4 days post-injection, the upward trend only became apparent at day 4 post-injection in groupers fed the NaB-supplemented diet. These observations thus tend to indicate that although $\mathrm{NaB}$ may not be effective in ameliorating the immunosuppression caused by high doses of 7S, it can, to a certain extent, delay the onset of disease caused by $V$. parahaemolyticus, thereby gaining valuable time for treatment. However, given that it was unclear how this resistance is regulated, we conducted the research described in the following section.

It is widely believed that the allergic responses to specific antibody-mediated humoral immunity and Tcell-mediated cellular immunity, which induce the production of serum-specific antibodies, occur primarily in the intestinal tract of fish ingesting $7 \mathrm{~S}^{18}$. As a typical pro-inflammatory cytokine, IL-1 $\beta$ is primarily produced by mononuclear macrophages, and can induce the activation of neutrophils and macrophages, stimulate the release of thromboxane and platelet-activating factor, increase the permeability of epithelial and endothelial cells, and induce intestinal inflammation. In the present study, we found that levels of IL$1 \beta$ in bL and bH group fish were significantly down- and upregulated, respectively. The lower levels of IL$1 \beta$ in the bL group conceivably indicate that low-dose $7 S$ can maintain normal serum immune responses by modifying IL-1 $\beta$ secretion, whereas the significantly elevated levels of IL-1 $\beta$ in the bH group, could indicate that at higher doses, $7 \mathrm{~S}$ may promote $\mathrm{CD} 4^{+} \mathrm{T}$-cell activation, proliferation, and differentiation by enhancing the abnormal secretion of IL-1 $\beta$, thereby exacerbating the inflammatory response ${ }^{19}$. We also observed that $\mathrm{NaB}$ supplementation of the grouper diet had the effect of significantly reducing serum IL$1 \beta$, thereby indicating that $\mathrm{NaB}$ may modulate serum immunity by inhibiting the abnormal secretion of IL$1 \beta$. In this regard, studies on rats have found that $\mathrm{NaB}$ can induce the overexpression of IL-1 $\beta$ by inhibiting the binding of $\mathrm{p} 65$ to the IL-1 $\beta$ promoter ${ }^{20}$. We accordingly speculate that a similar mechanism operates in the hybrid grouper.

TNF-a is a further key cytokine that plays role in mediating intestinal inflammatory processes ${ }^{21}$, and has been shown to exacerbate the progression of enteritis by inducing the release of pro-inflammatory factors 22. Consequently, determining changes in the levels of TNF-a is important with respect to monitoring inflammatory responses in the body. We found that a low dose of $7 \mathrm{~S}$ significantly reduced serum TNF-a levels in the hybrid grouper, whereas in contrast, a high dose had the effect of promoting the secretion of TNF-a. Similarly, in turbot, it has been found that supplementing feed with 4-8\% 7S increased the expression of TNF-a, which ultimately inhibited growth. These observations indicate that 7S has a regulatory effect on TNF-a secretion in both hybrid grouper and other fish. Indeed, it has previously been found that the percentage of $\mathrm{CD}^{+}$lymphocyte sub-populations in mice increases linearly with increasing $7 \mathrm{~S}$ levels, and thus we hypothesized that the elevation of TNF-a in the high-dose 7S group may be associated with an increase in $\mathrm{CD} 4^{+}$lymphocyte sub-populations in groupers ${ }^{23}$. We also found that supplementation of the grouper diet with $\mathrm{NaB}$ had the effect of reducing serum TNF-a to levels comparable with those observed in FM group fish, which we suspect could be attributable to the fact that $\mathrm{NaB}$ can inhibit TNF-a secretion by down-regulating LITAF expression ${ }^{24}$. This effect may also be 
associated with the fact that $\mathrm{NaB}$ inhibits mast cell activation and suppresses the release of mast cell inflammatory mediators.

Similarly to TNF-a, the type II interferon IFN-y play roles in immune and inflammatory responses. $7 \mathrm{~S}$ is treated by APC cells and the 7S soymetide is exposed, which binds to B- or T-cell-transformed immunoglobulins ${ }^{25}$. On the basis of the findings of the present study, it would appear that both low and high doses of 7S can promote the secretion of IFN- $y$, and we accordingly speculate that this may be closely related to the activation of $\mathrm{T}$ cells by $7 \mathrm{~S}$ as an antigenic protein. This assumption tends to be supported by our observations indicating that dietary supplementation with $\mathrm{NaB}$ was associated with a significant elevation in the levels of serum IFN- $\mathrm{N}$. NaB was also found to inhibit certain IFN- $\gamma$ functions by inhibiting the downstream signaling of IFN- $\gamma$ and thus the production of IFN- $\gamma$-induced factors ${ }^{26}$. We accordingly conjecture that, similar to humans, $\mathrm{NaB}$ can reduce the production of toxic substances in groupers by blocking the induction of IFN- $y$ on inflammatory cells. To examine this possibility, we conducted the experiments described in the following section.

Nitric oxide (NO), a free radical gas produced from l-arginine via the catalytic activity of nitric oxide synthase (NOS), plays an important role in host defense and inflammatory responses, and can interact with cytokines such as TNF- $a$ and IFN- $y$, thereby influencing the course of inflammatory responses ${ }^{27-29}$. Currently, however, there is a degree of controversy as to the mechanism whereby NO is regulated in the intestines ${ }^{27,30}$. In the present study, we found that NOS showed an elevated trend in the bL group and NO was significantly elevated in the fish in this group. A similar pattern was also observed in groupers in the $\mathrm{bH}$ group. Notably in this regard, we also detected significant increases and decreases in the levels of IFN$y$ and TNF- $a$ in the bL group, respectively, the former of which can enhance NO secretion by stimulating macrophages and inducing the production of nitric oxide synthase ${ }^{31}$. However, given that TNF-a showed an increase and decrease in the $\mathrm{bL}$ and $\mathrm{bH}$ groups, respectively, we were unable to determine whether TNF-a is involved in the regulation of NO production. Nevertheless, we speculate that either TNF-a is not involved at low doses of $7 \mathrm{~S}$ but is involved at high doses, or, alternatively, that TNF-a does not play a role in regulating the macrophage production of $\mathrm{NO}$ in the hybrid grouper. In addition, we observed that dietary supplementation with $\mathrm{NaB}$ maintained the significant upregulated production of IFN- $\gamma$, and resulted in the recovery of TNF-a to FM group levels. Correspondingly, NOS levels were significantly reduced, and NO showed a non-significant downward trend. Thus, taking into consideration the results obtained for both IFN- $\gamma$ and TNF-a, we are more inclined to believe that NaB downregulates NOS activity by inhibiting the release of TNF-a ${ }^{32}$, thereby reducing $\mathrm{NO}$ levels. The $\mathrm{ONOO}^{-}$is produced by the rapid binding of $\mathrm{NO}$ to oxygen radicals, which may be an important factor in cell damage, energy consumption, and cell death 33. We found that levels of $\mathrm{ONOO}^{-}$in the bL group were significantly downregulated, which contrasts with the elevated levels of NO in this group. Our observations tended to indicate that both IFN- $\gamma$ and IL-1 $\beta$ and TNF- $a$ are involved in the regulation of the NO-binding peroxide-generating step of $\mathrm{ONOO}^{-}$production. And from the analysis just presented, TNF- $a$ and IL-1 $\beta$ are very important in the regulation of $\mathrm{ONOO}^{-}$ generation ${ }^{34}$, particularly at high doses of $7 \mathrm{~S}$. We also found that $\mathrm{NaB}$ supplementation significantly reduced NOS levels, which may be related to the inhibition of NOS promoter-dependent transcriptional 
activity by $\mathrm{NaB}^{35}$. Excess $\mathrm{ONOO}^{-}$can adversely affect tissues primarily by damaging DNA, inactivating enzymes, and degrading mitochondria, and thus the downregulation of $\mathrm{ONOO}^{-}$in the $\mathrm{bH}-\mathrm{NaB}$ group may be related to the fact that $\mathrm{NaB}$ can enhance the provision of energy to cells ${ }^{36,37}$, thereby preventing the generation of $\mathrm{ONOO}^{-}$and thus preventing its negative effects.

As previously mentioned, the strong oxidizing properties of $\mathrm{ONOO}^{-}$and its interaction with cytokines such as TNF- $\alpha$ and IL-1 $\beta$ can induce cellular damage and thus disrupt the normal morphology of tissues. To better visualize the effects of $\mathrm{NO}$ and $\mathrm{ONOO}^{-}$on the intestinal tract of hybrid grouper, we prepared histomorphological sections of the hindgut, which is particularly susceptible to 7S-related disturbance (Periodic acid Schiff and Alcian Blue staining, AB-PAS). Intestinal development in fish can often reflect digestive absorption, and in this regard, plica height is typically used as an indicator of intestinal health. However, we would argue that plica height alone does not truly reflect the status of intestinal health. Therefore, as an alternative, we used the ratio of intestinal diameter to plica height (Id/Ph), which we believe to be a more reliable indicator of intestinal integrity. The larger this ratio, the greater is the space occupied by folds in the intestines, and thus the greater is the efficiency of absorption ${ }^{17}$. On the basis of the findings of the present study, we established that a high dose of 7S can significantly inhibited the development of plicae in the distal intestine of hybrid grouper, thereby reducing the absorptive area of the intestinal fold and thus the efficiency with which feed nutrients are absorbed. However, supplementing the grouper diet with $\mathrm{NaB}$ reversed the detrimental effects of high-dose 7, resulting in a reduction in the Id/Ph ratio, and significantly enhancing intestinal development. Similar results have also been obtained for the yellow drum (Nibea albiflora, Richardson) ${ }^{38}$.

The mucus secreted by the mucous cells of fish contains non-specific immunochemical substances, including lysozyme, transfer factors, chitin, and complementary substances, that are resistant to pathogenic microorganisms ${ }^{39}$. On the basis of AB-PAS staining, mucous cells can be classified into four types: I (pure red), II (pure blue), III (purple reddish), and IV (blue purple). From Fig. 2, it can be seen that most of the mucous cells in the distal intestine of hybrid grouper are type II cells. As an acidic mucous cell, the acidic mucus secreted by type II mucous cells plays an important role in regulating the transport of proteins and their residues, lubricating coarse feed, and increasing the viscosity of mucus and immune protection ${ }^{40}$. In the present study, we observed that the numbers of type II mucus cells in the intestines of $\mathrm{bL}$ and $\mathrm{bH}-\mathrm{NaB}$ group fish were not significantly different from those in the FM group, whereas in contrast, numbers were significantly reduced in the bH group, thereby indicating that a high dose of $7 \mathrm{~S}$ can significantly inhibit the formation of type II mucus cells and thus result in the reduced secretion of antimicrobial active substances. Conversely, supplementing the grouper diet with $\mathrm{NaB}$ was found to effectively increase the abundance of type II mucous cells, thereby enhancing the ability of the distal intestine of hybrid groupers to resist pathogenic microorganisms, and thus protecting the distal intestine.

Subsequent to the ingestion of antigenic proteins in feed, most is converted to amino acids and other small molecules. However, owing to the high molecular weight of $7 \mathrm{~S}$, a small fraction is not readily broken down and directly enters the lymphatic system via the intestinal mucosal spaces to induce an

Page $12 / 27$ 
immune response ${ }^{41}$. When the antigen re-enters the body from the intestines, it will cause intestinal mucosa damage and edema, leading to inflammation. In this regard, although numerous studies have examined the effects of antigenic proteins on the intestines of fish, few have investigated the root cause of the inflammatory effects.

The major histocompatibility complex (MHC) processes foreign antigens, generating antigenic peptide$\mathrm{MHC}$ molecular complexes, which are present in T cells and play an important role in inducing immune responses ${ }^{42}$. In this process, $\mathrm{MHC}$ II and $\mathrm{MHC}$ I present exogenous and endogenous antigens, respectively, and in the present study, we focused on the mechanisms associated with the former. In mammals, MHC II encapsulates foreign antigens within vesicular primary endosomes in response to an antigen signal. The principal genes involved in the MHC II antigen presentation process are GILT, AEP, and CTSB ${ }^{43,44}$, which function cooperatively to transport the primary endosomes to lysosomes for hydrolysis and antigen degradation. Thereafter, the complexes enter the Golgi apparatus for processing, and are thereby converted to antigenic peptide-MHC II molecular complexes, which activate CD4 T cells and produce corresponding immune responses ${ }^{45}$. In this regard, rather than binding directly to the promoter of the MHC II gene, CIITA has been shown to act by controlling MHC II gene transcription via a synergistic interaction with the essential transcription factors RFX5, CREB, and NFY ${ }^{46,47}$. In the present study, we found that the addition of a low dose of 7S promoted the synergistic association between CIITA and CREB1 and NFY to initiate the transcription of MHC II, thus presenting the foreign 7S antigenic protein (Fig. 7(a)). In contrast, we detected no significant changes in RFX5, and thus MHC I was not upregulated. Studies in patients with type III naked lymphocyte syndrome (BLS) have shown that the RFX complex is required for CIITA-mediated MHC I activity ${ }^{47}$, and it is speculated that this is also the case in hybrid grouper. In response to receiving the antigenic signal from 7S, MHC II forms an intranuclear body regulated by GILT, AEP, and CTSB, and is subsequent to processing within lysosomes and the Golgi system to form an antigenic peptide-MHC II molecular complex, which then transmits the signal to CD4 T cells to activate the immune response. In the distal intestine of hybrid grouper, AEP does not appear to be involved in transduction to nucleosomes, but rather promotes the transport of nucleosomes to lysosomes by upregulating GILT and downregulating CTSB, as has also been observed in mice ${ }^{48}$. However, the involvement of AEP appears to be related to the type of antigen detected by an organism, as AEP preferentially deals with viral antigens, as opposed to macromolecular protein antigens ${ }^{49}$. CD 4 T cells are activated upon receiving signals from the molecular complex, which in turn secrete cytokines with immunomodulatory properties. In combination with the results obtained from our examination of serumrelated immunity, these findings indicate that CD4 T cells and macrophages can enhance intestinal immunity in hybrid groupers by inhibiting the secretion of the pro-inflammatory factors IL-1 $\beta$ and TNF-a and promoting secretion of the anti-inflammatory factor IFN- $\gamma$.

The development of intestinal inflammation is generally accompanied by the abnormal expression of constituents of the PI3K/Akt/mTOR pathway ${ }^{50}$, the regulation of which by anti-nutritional factor-induced intestinal inflammation is currently incompletely understood. PI3K is a heterodimer composed of the regulatory subunit $\mathrm{p} 85$ and the catalytic subunit $\mathrm{p} 110$, the activities of which provide a signal for Akt, 
using PIP3 as a second messenger ${ }^{51}$. mTOR is an atypical serine/threonine protein kinase comprising two complexes, mTOR C1 and mTOR C2. The former consists of three core components, namely, mTOR, Raptor, and mLST8. mTOR is the catalytic subunit of the complex, whereas $\mathrm{mLST} 8$ is associated with the catalytic domain of $\mathrm{mTOR} \mathrm{C1}$, which stabilizes kinase activation, although is not the catalytic subunit of mTOR C1. mLST8 however, is associated with the catalytic domain of mTOR C1 that stabilizes kinase activation. Rictor does not seem to affect mTOR C1 activity ${ }^{52}$. mTOR C2 consists of three core components, namely, mTOR, Rictor, and mLST8, among which mTOR is a component of the catalytic subunit of the complex, as in $\mathrm{mTOR}$ C1. mTOR is a rapamycin-insensitive partner that can interact with Protor, although the physiological function of this interaction remains to be determined. $\mathrm{mLST} 8$ is a complex catalytic subunit of mTOR C2 ${ }^{53}$.

Akt also receives signals from mTOR $\mathrm{C} 2$, thereby promoting the expression of the core protein Rheb via the TSC1/TSC2 complex, which regulates mTOR $\mathrm{C}^{54}{ }^{54}$. On the basis of the low-dose $7 \mathrm{~S}$ results obtained in the present study, we established that in response to this treatment, Akt is not regulated by mTOR C2 or $\mathrm{PI} 3 \mathrm{~K}$, and does not promote Rheb expression via TSC2, thereby disrupting the regulation of mTOR C1. Moreover, in mTOR C1, TEL2 promotes the expression of 4E-BP2, although EIF4E is unaffected, whereas in mTOR C2, TEL2, and Sin 1 are likely to be the key components responsible for the upward modulation of RhoA. Furthermore, although the expression of TSC1 is elevated, in combination with Rheb, the regulation of mTOR C1 does not appear to be controlled by Akt. Given the aforementioned observations, we speculate that activation of the PI3K/Akt/mTOR pathway in response to low doses of $7 \mathrm{~S}$ is superficial and does not have a fundamental effect on key downstream proteins, with CD 4 T-cell activation playing a major role.

In response to supplementation with a high dose of 7S (Fig. 7(b)), MHC I synthesis is also upregulated via RFX5 activation, and a comparison with the response to low doses indicates that RFX5 is essential for $\mathrm{MHC}$ I regulation ${ }^{47}$. Subsequent to the formation of nuclear endosomes, it appears that GILT and AEP, although not GILT and CTSB, are involved in regulation. This observation indicates that the expression of AEP may be dependent not to the type of antigen, but rather the dose of antigen. In this case, following the activation of CD 4 T cells by the antigenic peptide-MHC II molecular complex, the secretion of IFN- $\gamma$ remains at high levels, and there are also significant increases in the expression of the pro-inflammatory factors IL-1 $\beta$ and TNF-a, thereby contributing to the development of intestinal inflammation. Interestingly in this regard, we also detected an upregulation of endogenous antigen presentation by MHC I, which we suspect may be associated with the elevated levels of body endogenous antigens induced by high doses of 7S. Likewise, we found that the PI3K/Akt/mTOR pathway was also fully activated in response to stimulation with high doses of 7S. Commencing with PI3K, which activates Akt by upregulating 3-PDK1, we found that IKKa was also upregulated, and although we did not examine the role played by NF-kB in the present study, we speculate that Akt may induce the transfer of NF-KB to the nucleus via IKKa. With respect to $\mathrm{mTOR}$ C1, the components depressor, Raptor, and $\mathrm{mLST} 8$ are activated, resulting in an $\mathrm{mTOR}$ C1-induced upregulation of 4E-BP1 and 4E-BP2, which in turn results in an increase in EIF4E secretion. Moreover, p70 S6K also increased the level of EIF4B via mTOR C1. Upregulation of elF-4E is often

Page $14 / 27$ 
considered a marker of early colon cancer, and it has also been reported that 4E-BP1 and 4E-BP2 limit the anti-inflammatory response of macrophages by inhibiting IL-10 ${ }^{55}$. Thus, high doses of 7S-induced intestinal inflammation in the hybrid grouper may be associated with elevated levels of 4E-BP1 and 4EBP2 and a suppression of the anti-inflammatory effects of macrophages. In addition, the expression of TSC1 is elevated, thereby in turn promoting the expression of Rheb, which relays signals from Akt to mTOR C1. Furthermore, with respect to $\mathrm{mTOR}$ C2, mLST8, depressor, and RICTOR are also activated, thereby elevating the levels of SGK1, which is involved extensively in the regulation of inflammation, and not only induces cardiac inflammation by activating the NLRP3 inflammasome ${ }^{56}$ but can also exacerbate inflammation by stimulating NF-kB ${ }^{57}$.

As a common feed additive used to supplement the diets of livestock, poultry, and aquatic animals, $\mathrm{NaB}$ can be hydrolyzed in the gastrointestinal tract to form butyric acid, which provides a source of energy to the intestinal epithelial cells, and contributes to the repair of damaged intestinal mucosa and strengthening intestinal immunity. However, the mechanisms whereby $\mathrm{NaB}$ influences intestinal repair have yet to be determined. In the present study, we found that supplementation of feed containing a high dose of $7 \mathrm{~S}$ with appropriate levels of $\mathrm{NaB}$ facilitated the transcription of $\mathrm{MHC}$ I and $\mathrm{MHC}$ II via the upregulated expression of CREB1 and downregulation of NFY, and that MHC II expression was maintained at a high level (Figure(c)). These observation thus tend to indicate that dietary supplementation with $\mathrm{NaB}$ has the effect of enhancing the efficiency of antigenic protein presentation and processing in hybrid groupers. In addition, a concomitant reduction in the expression of MHC I may be indicative of a reduction in the severity of the inflammatory response compared with that seen in groupers receiving a high dose of $7 \mathrm{~S}$ in the absence of $\mathrm{NaB}^{58}$. In addition GILT and AEP are inhibited during the development of nucleosomes, which subsequently migrate to the lysosomes and Golgi apparatus. There is also a reduction in expression of the pro-inflammatory factors IL-1 $\beta$ and TNF- $a$, thereby indicating a weakening of the inflammatory response. Although PI3K expression was also upregulated, the expression of 3-PDK1 and Akt remained at levels similar to those observed in groupers receiving a high dose of 7S. However, we found that the key transcription factor IKKa, which is transduced by Akt to NF-kB, was suppressed. On the basis of these observations, we thus speculate that $\mathrm{NaB}$ supplementation could suppress the activation of NF-KB ${ }^{59}$, although we did not verify this assumption in the present study. With regards to the mTOR C1 complex, we noted an inhibition of mLST8 and Raptor, which in turn downregulated the expression and secretion of 4E-BP1 and EIF4B, and although the expression of p70 S6K was maintained at a high level, the formation of the downstream product EIF4B was inhibited.

Collectively, these findings indicate that the protective effect of $\mathrm{NaB}$ on the hindgut of hybrid grouper is closely associated with small molecule proteins downstream of $\mathrm{mTOR}$ C1, such as EIF4E. Although TSC1 showed high expression, that of Rheb was significantly reduced, and taken together with the results obtained for groupers receiving feed supplemented with high and low doses of 7S, we speculate that there are probably alternative mechanisms regulating the pathway from Akt to mTOR C1. With respect to mTOR C2, we established that the downregulated expression of $\mathrm{mLST} 8$ and Sin 1 and upregulation of 
PRR5 promoted an increase in the expression of RhoA and PKC, and that SGK1 concomitantly remained at high levels. RhoA and PKC are generally considered to function as signal molecules associated with the inflammatory response, and are accordingly also commonly used as targets to suppress inflammation ${ }^{60,61}$. Thus, $\mathrm{NaB}$ does not appear to alleviate intestinal inflammation via mTOR C2, but rather exacerbates the progression of intestinal inflammation.

\section{Conclusion}

In the present study, we demonstrated that low and high doses of $\beta$-conglycinin (7S) significantly enhanced and reduced the weight gain rate, specific growth rate, and feed utilization of hybrid grouper, respectively. However, we found that supplementation of the grouper diet with sodium butyrate was effective in relieving the growth inhibition caused by high doses of 7S. High-dose 7S was shown to disrupt the development of distal intestinal plica, suppress the density of distal intestinal type II mucus cells, and enhance the synthesis of $\mathrm{NO}$, NOS, and $\mathrm{ONOO}^{-}$associated with intestinal injury, thereby exacerbating the process of distal intestinal inflammation. The enhancement of immune responses in the distal intestine of hybrid grouper receiving a low dose of $7 \mathrm{~S}$ appears to be associated primarily with the activation of CD4 T cells, which promotes the release of the anti-inflammatory factor IFN- $y$ and reduces that of TNF- $a$ and IL-1 $\beta$. However, this does not appear to be mediated via by the PI3K/Akt/mTOR pathway. In contrast, this pathway was found to be fully activated upon stimulation with high doses of 7S. Within this pathway, Akt activates mTOR C1 via the promotion of Rheb expression, which in turn enhances the expression of the downstream pro-inflammatory small molecule proteins EIF4E and 4E-BP2, whereas Akt also upregulates SGK1 expression via mTOR C2. These dual effects synergistically contribute to exacerbating inflammation in the distal intestine of hybrid grouper. Furthermore, we established that sodium butyrate relieves intestinal inflammation by inhibiting the expression of the inflammatory-related small molecule proteins 4E-BP2 and EIF4B downstream of mTOR C1. However, in the case of mTOR C2, we found that sodium butyrate does not protect the distal intestine by inhibiting RhoA, PKC, and SGK1, but instead further intensified intestinal inflammation by upregulating RhoA and PKC. In combination with the results obtained for growth performance and histopathological analyses, our findings indicate PI3K/Akt/mTOR C1 and PI3K/Akt/mTOR C2 play major roles in mediating 7Sinduced immune responses in the distal intestine of hybrid grouper, and with respect to the mechanisms underlying the ameliorative effect of sodium butyrate, we suspect that MTOR C1 plays a more important role than $\mathrm{mTOR}$ C2.

\section{Declarations}

\section{Data availability}

All datasets generated during and/or analyzed during the current study are available from the corresponding author on reasonable request. 


\section{Acknowledgments}

We would like to thank the Key Laboratory of Control for Disease of Aquatic Economic Animals of Guangdong Higher Education Institutes (Zhanjiang, China) for kindly providing the Vibrio parahaemolyticus strain used in this study.

\section{Author contributions}

All indicated authors have actively contributed to this study. The contributions of the co-authors are as follows: H. Y. Liu and B. P. Tan designed the study; B. Yin conducted the study and analyzed the data; X. H. Dong participated in the interpretation of results; B. Yin wrote the manuscript; Q. H. Yang, S. Y. Chi, and S. Zhang purchased the reagent supplies; $H$. Y. Liu revised the manuscript. All authors have read and approved the final version of the manuscript.

\section{Competing interests}

The authors declare that they have no competing interests.

\section{Funding}

This work was supported by the National Key R\&D Program of China (2019YFD0900200) and the National Natural Science Foundation of China (No.31772864) and the Natural Science Foundation of Guangdong Province (2018A030313154\&2020A1515011129).

\section{References}

1. Jiang, S. T. et al. Optimal dietary protein level and protein to energy ratio for hybrid grouper

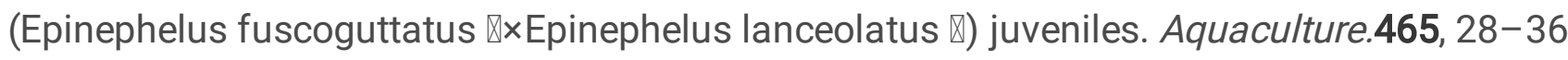
https://doi.org/10.1016/j.aquaculture.2016.08.030 (2016).

2. Firdaus, R. F., Lim, L. S., Kawamura, G. \& Shapawi, R. Assessment on the acceptability of hybrid

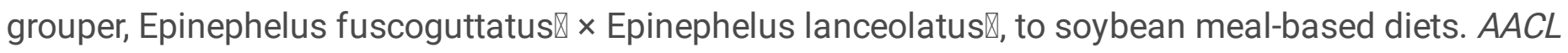
Bioflux.9, 284-290 (2016).

3. Zhang, W., Tan, B., Ye, G., Wang, J. \& Zhang, H. Identification of potential biomarkers for soybean meal-induced enteritis in juvenile pearl gentian grouper, Epinephelus lanceolatus $\mathbb{X} \times$ Epinephelus fuscoguttatus $\varangle$. Aquaculture.512, 734337 https://doi.org/10.1016/j.aquaculture.2017.05.008 (2019).

4. Li, Y. X., Yang, P., Zhang, Y. J., Ai, Q. H. \& Xu, W. Effects of dietary glycinin on the growth performance, digestion, intestinal morphology and bacterial community of juvenile turbot, Scophthalmus maximus L. Aquaculture.479, 125-133 https://doi.org/10.1016/j.aquaculture.2017.05.008 (2017). 
5. Hao, Y., Zhan, Z. F., Guo, P. F., Piao, X. S. \& Li, D. F. Soybean beta-conglycinin-induced gut hypersensitivity reaction in a piglet model. Arch. Anim. Nutr.63, 188-202 https://doi.org/10.1080/17450390902860026 (2009).

6. Xu, D. D. et al. Soybean $\beta$-conglycinin caused intestinal inflammation and oxidative damage in association with NF-KB, TOR and Nrf2 in juvenile grass carp (Ctenopharyngodon idella): varying among different intestinal segments. Fish Shellfish Immunol.95, 105-116 https://doi.org/10.1016/J.FSI.2019.10.021 (2019).

7. Hamer, H. M. H. et al. Review article: the role of butyrate on colonic function. Aliment. Pharm. Ther.27, 104-119 https://doi.org/10.1111/J.1365-2036.2007.03562.X (2007).

8. Liu, W. S. et al. Effects of dietary microencapsulated sodium butyrate on growth, intestinal mucosal morphology, immune response and adhesive bacteria in juvenile common carp (Cyprinus carpio) prefed with or without oxidised oil. Br. J. Nutr.112, 15-29 https://doi.org/10.1017/S0007114514000610 (2014).

9. Livak, K. J. \& Schmittgen, T. D. Analysis of relative gene expression data using real-time quantitative PCR and the 2(-Delta Delta C(T)) method. Methods.25, 402-408 https://doi.org/10.1006/meth.2001 (2001).

10. Yin, B. et al. Preliminary study of mechanisms of intestinal inflammation induced by plant proteins in juvenile hybrid groupers (खEpinephelus fuscoguttatus $\times \mathrm{E}$. lanceolatu). fish \& shellfish immunology.106, 341-356 https://doi.org/10.1016/J.FSI.2020.07.026 (2020).

11. Guo, X. Z. et al. The growth-promoting effect of dietary nucleotides in fish is associated with an intestinal microbiota-mediated reduction in energy expenditure. J. Nutr.147, 781-788 https://doi.org/10.3945/jn.116.245506 (2017).

12. Zhang, J. X. et al. Soybean $\beta$-conglycinin induces inflammation and oxidation and causes dysfunction of intestinal digestion and absorption in fish. Plos One.8, e58115 https://doi.org/10.1371/journal.pone.0058115 (2014).

13. Zhou, Q. C., Mai, K. S., Tan, B. P. \& Liu, Y. J. Partial replacement of fishmeal by soybean meal in diets for juvenile cobia (Rachycentron canadum). Aquacult. Nutr.11, 175-182 https://doi.org/10.1111/J.1365-2095.2005.00335.X (2015).

14. Li, Y. X. et al. Dietary soya allergen $\beta$-conglycinin induces intestinal inflammatory reactions, serumspecific antibody response and growth reduction in a carnivorous fish species, turbot Scophthalmus maximus L. Aquac. Res.48, 4022-4037 https://doi.org/10.1111/ARE.13224 (2017).

15. Gu, M., Bai, N., Zhang, Y. Q. \& Krogdahl, S. Soybean meal induces enteritis in turbot Scophthalmus maximus at high supplementation levels. Aquaculture.464, 286-295 https://doi.org/10.1016/j.aquaculture.2016.06.035 (2016).

16. He, Y. F. et al. Integrative transcriptomic and small RNA sequencing reveals immune-related miRNAmRNA regulation network for soybean meal-induced enteritis in hybrid grouper, Epinephelus

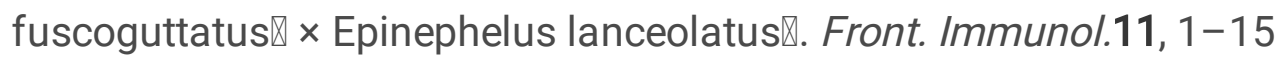
https://doi.org/10.3389/FIMMU.2020.01502 (2020). 
17. Liu, M. M. et al. Dietary supplementation of sodium butyrate may benefit growth performance and intestinal function in juvenile grass carp (Ctenopharyngodon idellus). Aquac. Res.48, 4102-4111 https://doi.org/10.1111/ARE.13230 (2017).

18. O"Donnell, G. B., Reilly, P., Davidson, G. A. \& Ellis, A. E. The uptake of human gamma globulin incorporated into poly (D,L-lactide-co-glycolide) microparticles following oral intubation in Atlantic salmon, Salmo salar L. Fish Shellfish Immunol.6, 507-520 https://doi.org/10.1006/fsim.1996.0048 (1996).

19. Sun, H., Liu, X., Wang, Y. Z., Liu, J. X. \& Feng, J. Allergen-specific immunoglobulin, histamine and Tcell responses induced by soybean glycinin and $\beta$-conglycinin in BALB/c mice of oral sensitisation. Food Agr. Immunol.24, 489-501 https://doi.org/10.1080/09540105.2012.730501 (2013).

20. Zhong, X., Savidge, T., Cong, Y. \& Li, Q. PTH-073Severe neonatal inflammation epigenetically sensitises the colonic epithelium for exacerbated il-1B activation. Gut.66, A242https://doi.org/10.1136/gutjnl-2017-314472.472 (2017).

21. Parker, A. et al. Elevated apoptosis impairs epithelial cell turnover and shortens villi in TNF-driven intestinal inflammation. Cell Death Dis.10, 108-108 https://doi.org/10.1038/S41419-018-1275-5 (2019).

22. Lang, A. et al. Allicin inhibits spontaneous and TNF-a induced secretion of proinflammatory cytokines and chemokines from intestinal epithelial cells. Clin. Nutr.23, 1199-1208 https://doi.org/10.1016/j.clnu.2004.03.011 (2004).

23. Fei, G. P., Piao, X. S., Ou, D. Y., Li, D. F. \& Hao, Y. Characterization of the antigenic specificity of soybean protein $\beta$-conglycinin and its effects on growth and immune function in rats.Arch. Anim. Nutr.61,189-200, doi:doi: 10.1080/17450390701318358.

24. Han, F. L. et al. Effects of glycinin and $\beta$-conglycinin on growth performance and intestinal health in juvenile Chinese mitten crabs (Eriocheir sinensis). Fish Shellfish Immun.84, 269-279 https://doi.org/10.1016/j.fsi.2018.10.013 (2018).

25. Tsuruki, T. et al. Soymetide, an immunostimulating peptide derived from soybean $\beta$-conglycinin, is an fMLP agonist. Febs Lett.540, 206-210 https://doi.org/10.1016/S0014-5793(03)00265-5 (2003).

26. Kitamura, K. et al. Sodium butyrate blocks interferon-gamma (IFN-gamma)-induced biosynthesis of MHC class III gene products (complement $\mathrm{C} 4$ and factor $\mathrm{B}$ ) in human fetal intestinal epithelial cells. Clin. Exp. Immunol.118, 16-22 https://doi.org/10.1046/J.1365-2249.1999.01004.X (2010).

27. Kubes, P. \& Mccafferty, D. M. Nitric oxide and intestinal inflammation. Am. J. Med.109, 150-158 https://doi.org/10.1016/S0002-9343(00)00480-0 (2000).

28. Kim, M. K., Kim, K. B., Kim, H. S. \& Lee, B. M. Alternative skin sensitization prediction and risk assessment using proinflammatory biomarkers, interleukin-1 beta (IL-1 $\beta$ ) and inducible nitric oxide synthase (iNOS). J. Toxicol. Env. Heal. A.82, 361-378 https://doi.org/10.1080/15287394.2019.1609183 (2019).

29. Nakanishi, Y., Sato, T., Takahashi, K. \& Ohteki, T. IFN-y-dependent epigenetic regulation instructs colitogenic monocyte/macrophage lineage differentiation in vivo. Mucosal Immunol.11, 871-880 
https://doi.org/10.1038/mi.2017.104 (2018).

30. Anand, R. J., Rippel, C. A., Leaphart, C. L., Li, J. \& Hackam, D. J. Activated macrophages inhibit enterocyte gap junctions during intestinal inflammation through the release of nitric oxide. J. Surg. Res.130, 227 (2006).

31. Nieto-Patlán, E. et al. Valproic acid promotes a decrease in mycobacterial survival by enhancing nitric oxide production in macrophages stimulated with IFN-y. Tuberculosis.114, 123-126 https://doi.org/10.1016/J.TUBE.2018.12.007 (2019).

32. Liu, J., Chang, G. J., Huang, J., Wang, Y. \& Ma, N. N. Sodium butyrate inhibits the inflammation of lipopolysaccharide-induced acute lung injury in mice by regulating the toll-like receptor 4 /nuclear factor KB signaling pathway. J. Agr. Food Chem.67, 1674-1682 https://doi.org/10.1021/ACS.JAFC.8B06359 (2019).

33. Ródenas, J., Mitjavila, M. T. \& Carbonell, T. Simultaneous generation of nitric oxide and superoxide by inflammatory cells in rats. Free Radical Bio. Med.18, 869-875 https://doi.org/10.1016/08915849(94)00215-6 (1995).

34. Neumann, P. et al. Peroxynitrite mediates TNF-induced endothelial barrier dysfunction and nitration of actin. AM. J. Physiol-Lung C.290, L674-L684 https://doi.org/10.1152/AJPLUNG.00391.2005 (2006).

35. Sasahara, Y. et al. Suppression of promoter-dependent transcriptional activity of inducible nitric oxide synthase by sodium butyrate in colon cancer cells. Cancer Lett.177, 155-161 https://doi.org/10.1016/S0304-3835(01)00778-9 (2002).

36. Liu, J. D. et al. Evaluation of encapsulated sodium butyrate on growth performance, energy digestibility, gut development, and Salmonella colonization in broilers. J. Poult. Sci.96, 3638-3644 https://doi.org/10.3382/ps/pex174 (2017).

37. Fang, W. J., Xue, H. L., Chen, X., Chen, K. \& Ling, W. H. Supplementation with sodium butyrate modulates the composition of the gut microbiota and ameliorates high-fat diet-induced obesity in mice. J. Nutr.149, 747-754 https://doi.org/10.1093/JN/NXY324 (2019).

38. Wu, X., Wang, L. G., Xie, Q. P. \& Tan, P. Effects of dietary sodium butyrate on growth, diet conversion, body chemical compositions and distal intestinal health in yellow drum (Nibea albiflora, Richardson). Aquac. Res.51, 69-79 https://doi.org/10.1111/ARE.14348 (2020).

39. Bosi, G., DePasquale, J. A., Carosi, A., Lorenzoni, M. \& Dezfuli, B. S. Protective responses of intestinal mucous cells in a range of fish-helminth systems. J. Fish Dis.40, 1001-1014 https://doi.org/10.1111/JFD.12576 (2017).

40. Gui, K. J., Park, J. J., Lee, J. W. \& Lee, J. S. Microscopic anatomy of foot of the spiny top shell, Batillus cornutus (lightfoot, 1786) (Gastropoda: Turbinidae). J. Shellfish Res.25, 1071-1077 10.2983/0730-8000(2006)25[1071:MAOFOT]2.0.CO;2 (2007).

41. Chen, F. et al. Soybean-derived [Beta]-conglycinin affects proteome expression in pig intestinal cells in vivo and in vitro. J. Anim. Sci.89, 743-753 https://doi.org/10.2527/jas.2010-3146 (2011). 
42. Bremberg, N. Exploring the external dimension of european cooperation in the area of internal security: the case of euro-mediterranean civil protection. J. Immunol. Methods.169, 273-285 https://doi.org/10.1016/0022-1759(94)90271-2 (2018).

43. Ganesan, A. P. V., Miller, M. A. \& Eisenlohr, L. C. Endogenously-processed influenza epitopes intersect with the classical MHCIl presentation pathway via the GILT endosomal reductase (APP2P.104).J. Immunol.192 (2015).

44. Maehr, R. et al. Asparagine endopeptidase is not essential for Class II MHC antigen presentation but is required for processing of Cathepsin L in mice. J. Immunol.174, 7066-7074 https://doi.org/10.4049/jimmunol.174.11.7066 (2005).

45. Lvaro-Benito, M., Morrison, E., Ebner, F., Abualrous, E. T. \& Freund, C. Distinct editing functions of natural HLA-DM allotypes impact antigen presentation and CD4 + T cell activation. Cell. Mol. Immunol.17, 133-142 https://doi.org/10.1038/s41423-018-0181-1 (2018).

46. Wong, D. et al. Genomic mapping of the MHC transactivator CIITA using an integrated ChIP-seq and genetical genomics approach. Genome Biol.15, 494-494 https://doi.org/10.1186/S13059-014-0494Z (2014).

47. Gobin, S. J. P., Peijnenburg, A., Eggermond, M. V., Zutphen, M. V. \& Elsen, P. J. V. D. The RFX complex is crucial for the constitutive and CIITA-mediated transactivation of MHC class I and beta2microglobulin genes. Immunity.9, 531-541 https://doi.org/10.1016/S1074-7613(00)80636-6 (1998).

48. Testa, J. S. Elucidation of MHC class Il-restricted antigen processing pathways using influenza virus epitopes Doctoral thesis, Thomas Jefferson University(2010).

49. Watts, C., Matthews, S. P., Mazzeo, D., Manoury, B. \& Moss, C. X. Asparaginyl endopeptidase: case history of a class II MHC compartment protease. Immunol. Rev.207, 218-228 https://doi.org/10.1111/J.0105-2896.2005.00312.X (2005).

50. Kim, H. et al. Mango polyphenolics reduce inflammation in intestinal colitis-involvement of the miR126/PI3K/AKT/mTOR axis in vitro and in vivo. Mol. Carcinogen.56, 197-207 https://doi.org/10.1002/MC.22484 (2016).

51. Thapa, N., Choi, S., Tan, X., Wise, T. \& Anderson, R. A. Phosphatidylinositol phosphate 5-kinase IY and $\mathrm{PI} 3 \mathrm{~K} / \mathrm{Akt}$ signaling couple to promote oncogenic growth. J. Biol. Chem.290, 1-23 https://doi.org/10.1074/jbc.M114.596742 (2015).

52. Lang, S. A., Moser, C., Hackl, C., Fichtner-Feigl, S. \& Stoeltzing, O. Abstract 299: Blockade of Rictor impairs mTORC1 inhibitor-induced upregulation of IGF-IR and Her2 expression in human gastrointestinal cancer cells. Cancer Res.70, 299-299 https://doi.org/10.1158/1538-7445.AM10299 (2010).

53. Rosner, M. \& Hengstschlager, M. Cytoplasmic and nuclear distribution of the protein complexes mTORC1 and mTORC2: rapamycin triggers dephosphorylation and delocalization of the mTORC2 components rictor and sin1. Hum. Mol. Genet.17, 2934-2948 https://doi.org/10.1093/HMG/DDN192 (2008). 
54. Hsieh, C. T., Chuang, J. H., Yang, W. C., Yin, Y. \& Lin, Y. S. Ceramide inhibits insulin-stimulated Akt phosphorylation through activation of Rheb/mTORC1/S6K signaling in skeletal muscle. Cell.

Signal.26, 1400-1408 (2014).

55. William, M. et al. elF4E-Binding Proteins 1 and 2 Limit Macrophage Anti-Inflammatory Responses through Translational Repression of IL-10 and Cyclooxygenase-2. J. Immunol.200, 4102-4116 https://doi.org/10.4049/JIMMUNOL.1701670 (2018).

56. Gan, W. Q. et al. The SGK1 inhibitor EMD638683, prevents Angiotensin Il-induced cardiac inflammation and fibrosis by blocking NLRP3 inflammasome activation. BBA-Biomembranes.1864, 1-10 https://doi.org/10.1016/J.BBADIS.2017.10.001 (2017).

57. Wu, X. Q., Jiang, W., Wang, X. L., Zhang, C. \& Tian, Z. L. SGK1 enhances Th9 cell differentiation and airway inflammation through NF-kB signaling pathway in asthma. Cell Tissue Res. 1-12 https://doi.org/10.1007/S00441-020-03252-3 (2020).

58. Wu, Q. H. et al. Replication of human cytomegalovirus in cells deficient in $\beta 2$-microglobulin gene expression. J. Gen. Virology.75, 2755-2759 https://doi.org/10.1099/0022-1317-75-10-2755 (1994).

59 . $\mathrm{Si}, \mathrm{J}$. J. et al. Sodium butyrate $(\mathrm{NaB})$ could rescue memory reconsolidation in the presence of IKK inhibition with SSZ. Plos One.g006, 0043973 https://doi.org/10.1371/journal.pone.0043973.g006 (2015).

60. Fard, M. A., Ebrahimi, K. B. \& Miller, N. R. RhoA activity and post-ischemic inflammation in an experimental model of adult rodent anterior ischemic optic neuropathy. Brain Res.1534, 76-86 (2013).

61. Kong, L. et al. PKC $\beta$ promotes vascular inflammation and acceleration of atherosclerosis in diabetic ApoE null mice. Arterioscl. Throm. Vas.33, 1779-1787 https://doi.org/10.1161/ATVBAHA.112.301113 (2013).

\section{Figures}
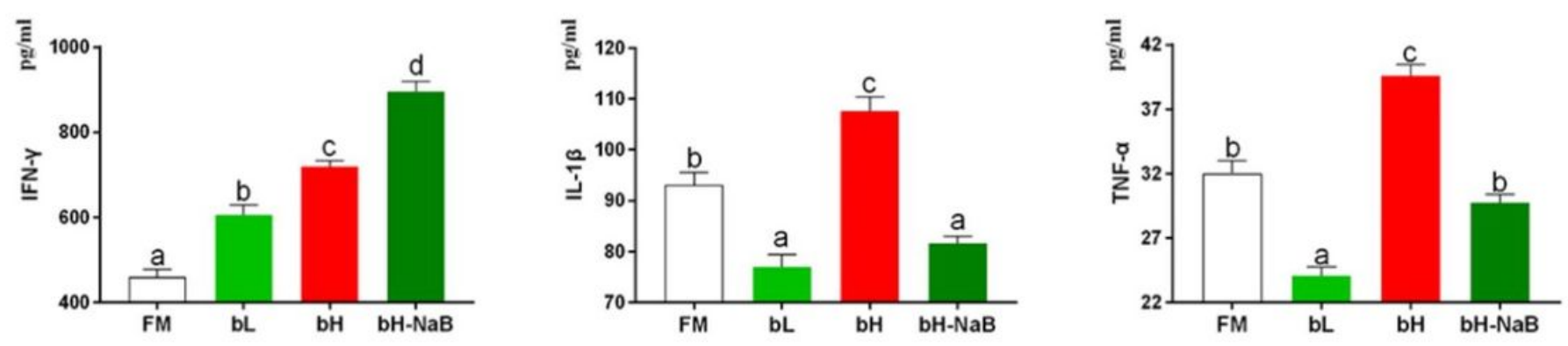

\section{Figure 1}


Serum immune indexes of juvenile hybrid groupers (Epinephelus fuscoguttatus $\nabla \times E$. lanceolatus $\varangle$ ) fed experimental diets for 8 weeks. Values are the means $\pm S E(n=4)$; Significance was evaluated by oneway ANOVA followed by Tukey multiple range tests. FM, control diet; bL, containing $1.5 \% 7 \mathrm{~S}$ diet, bH, containing $6 \% 7 \mathrm{~S}$ diet, $\mathrm{bH}-\mathrm{NaB}$, containing $6 \% 7 \mathrm{~S}$ and $0.13 \% \mathrm{NaB}$ diet. IFN- $\gamma$, interferon-gamma; IgE, immunoglobulin E; IL-1 $\beta$, interleukin-1 beta; TNF-a, tumor necrosis factor-alpha. a, b, c, dMean values denoted by different superscripted letters differ significantly at the $\mathrm{P}<0.05$ level.

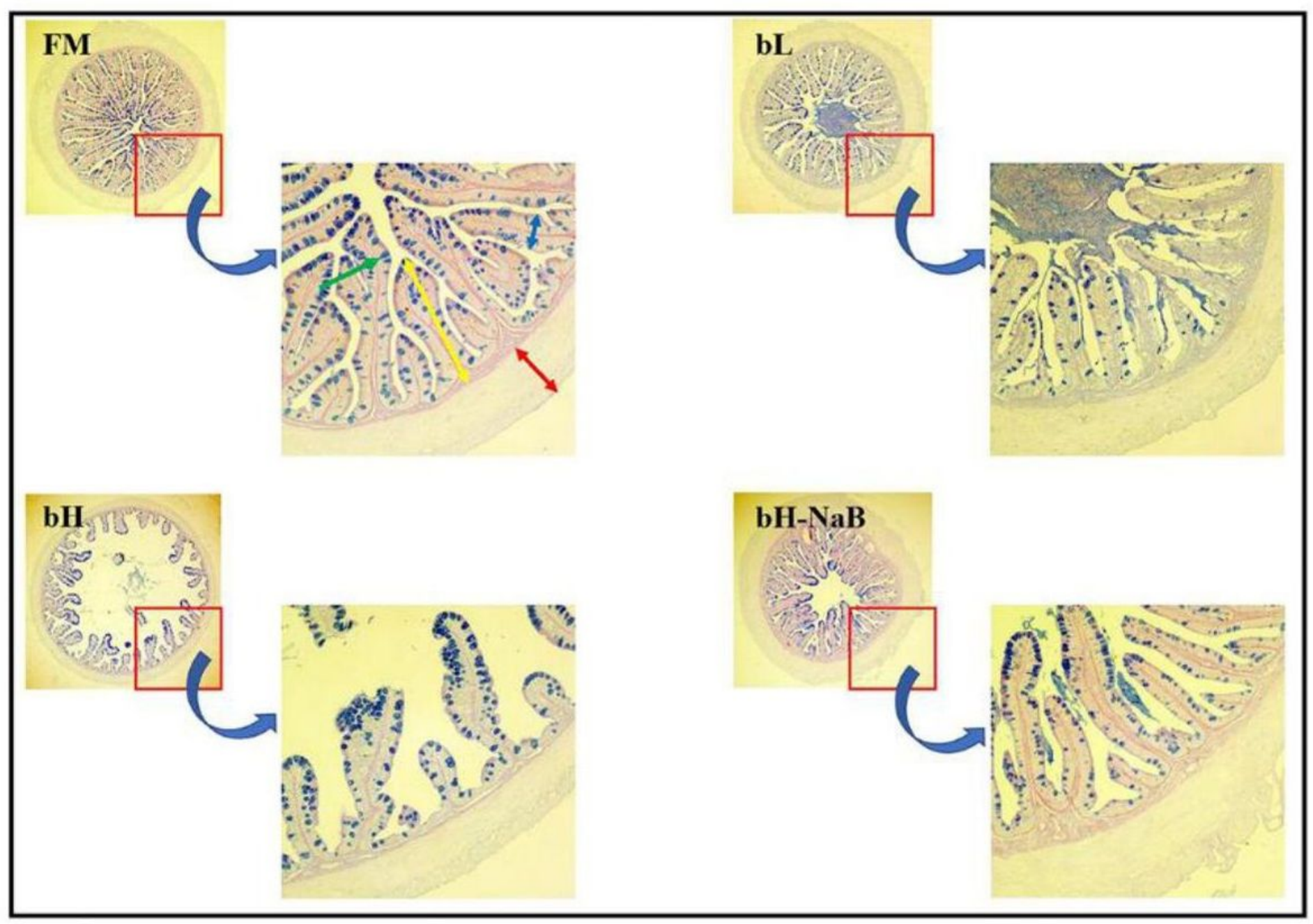

Figure 2

Distal intestinal histological evaluation based Alcian Blue-Periodic acid-Shiff staining of distal intestine

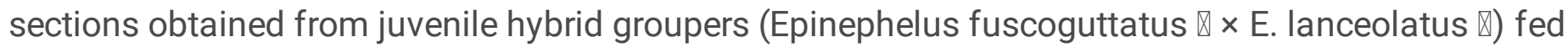
experimental diets for 8 weeks. Green arrow: type II mucous cell; red arrow: muscle thickness; yellow arrow: plica height: blue arrow: plica width. 

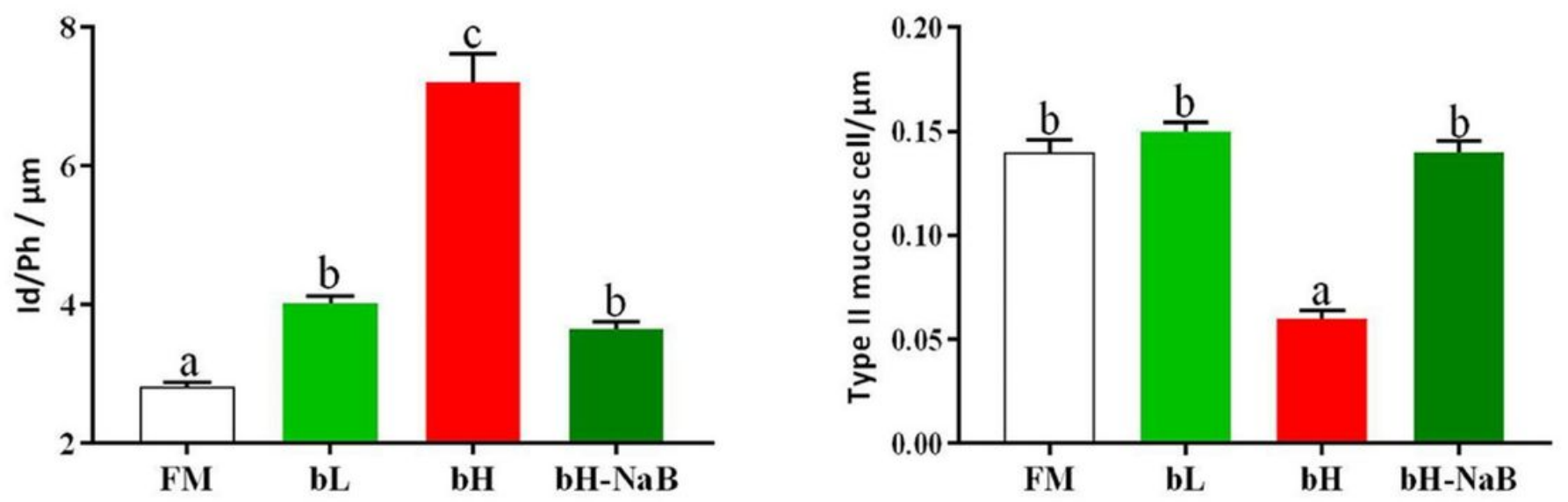

Figure 3

Distal intestinal histopathological observation of juvenile hybrid groupers (Epinephelus fuscoguttatus $\mathbb{x} \times$ E. lanceolatus $\triangle)$ fed experimental diets for 8 weeks $(P<0.05)$. a, b, cMean values denoted by different superscripted letters differ significantly at the $\mathrm{P}<0.05$ level.
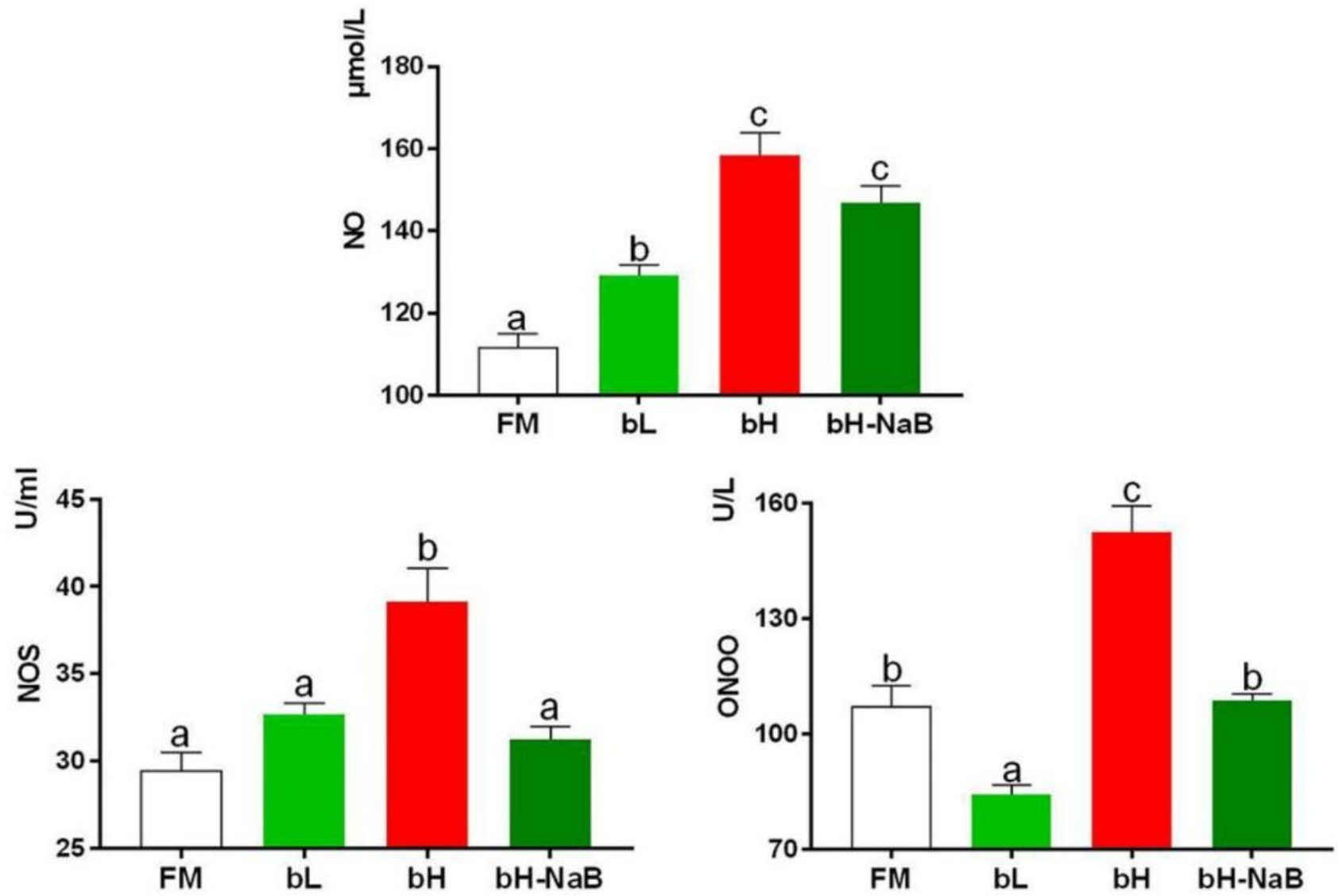

Figure 4 
Distal intestinal damage indicators of juvenile hybrid groupers (Epinephelus fuscoguttatus $\square \times \mathrm{E}$. lanceolatus $\mathbb{Q}$ ) fed the experimental diets for 8 weeks $(P<0.05)$. a, b, cMean values denoted by different superscripted letters differ significantly at the $\mathrm{P}<0.05$ level.
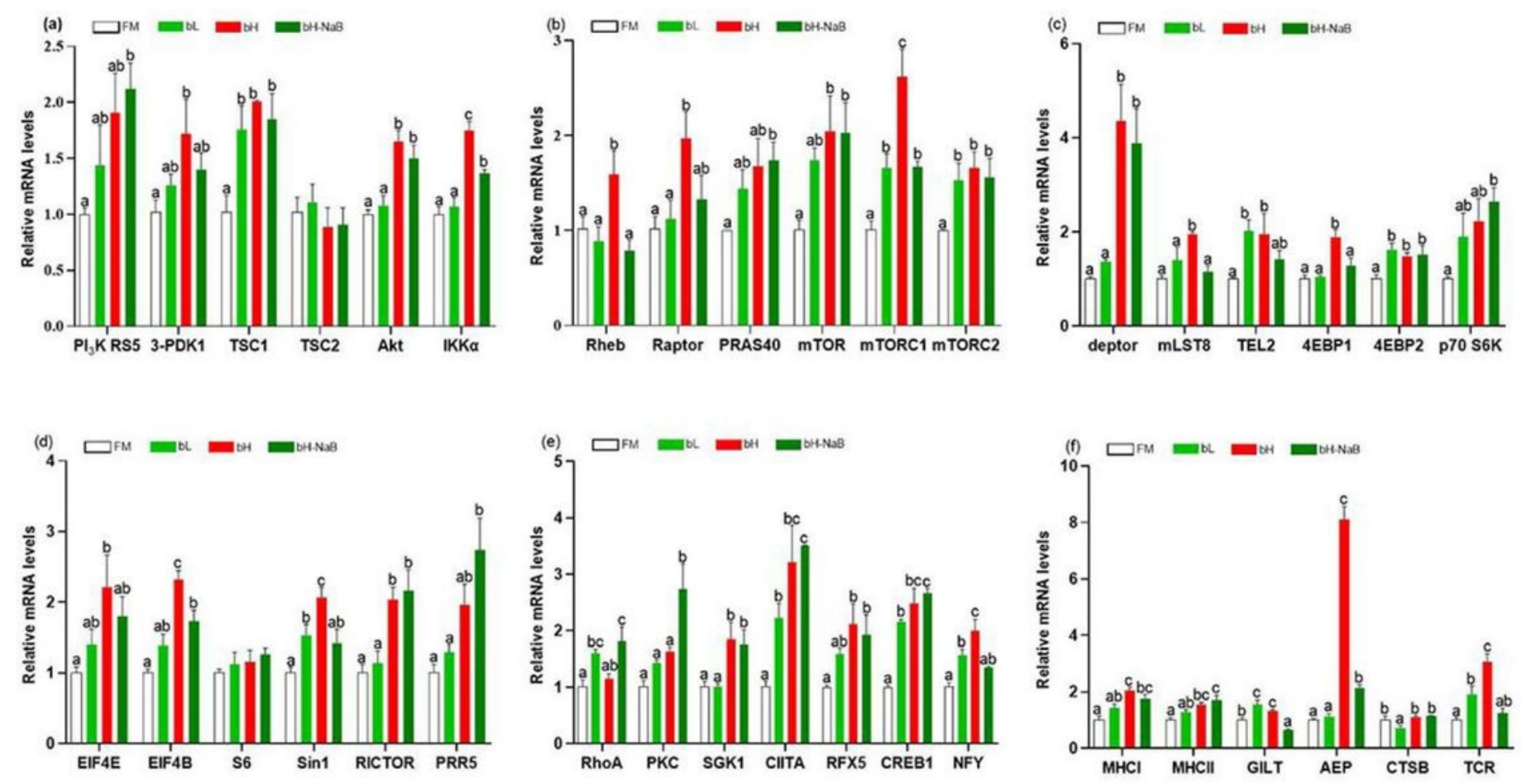

\section{Figure 5}

Distal intestinal antigen processing and presentation-related gene expression in juvenile hybrid groupers (Epinephelus fuscoguttatus $\square \times E$. lanceolatus $\nabla$ ) fed the experimental diets for 8 weeks. Results are represented as the means \pm SE $(n=4)$. PI3K RS5, phosphatidylinositol 3-kinase regulatory subunit 5; 3PDK1, 3-phosphoinositide dependent kinase-1; TSC1, tuberous 1; TSC2, tuberous 2; Akt, serine/threonineprotein kinase; IKKa, inhibitor of nuclear factor kappa-B kinase subunit a; Rheb, Ras homolog enriched in the brain; Raptor, regulatory associated protein of mTOR; PRAS40, proline-rich Akt1 substrate 1; mTOR, mammalian target of rapamycin; mTOR C1, mammalian target of rapamycin complex 1; mTOR C2, mammalian target of rapamycin complex 2; deptor, DEP domain-containing mTOR-interacting protein; $\mathrm{mLST} 8$, target of rapamycin complex subunit Ist8; TEL2, telomere length regulation protein; 4EBP1, eukaryotic translation initiation factor $4 \mathrm{E}$ binding protein 1 ; $4 \mathrm{EBP} 2$, eukaryotic translation initiation factor $4 \mathrm{E}$ binding protein 2; p70 S6K, ribosomal protein $\mathrm{S} 6$ kinase $\beta 1$; EIF4E, translation initiation factor $4 \mathrm{E}$; EIF4B, translation initiation factor 4B; S6, small subunit ribosomal protein S6; Sin1, target of rapamycin complex 2 subunit; RICTOR, rapamycin-insensitive companion of mTOR; PRR5, proline-rich protein 5; RhoA, Ras homolog gene family, member A; PKCa, protein kinase C a; SGK1, serum/glucocorticoidregulated kinase 1 ; CIITA, major histocompatibility complex class II trans-activator; RFX5, regulatory factor X5; CREB1, cyclic AMP-responsive element-binding protein 1; NFY, nuclear transcription factor $Y$ subunit a; MHC I, major histocompatibility complex class I antigen; MHC II, major histocompatibility 
complex class II antigen; GILT, gamma-interferon-inducible-lysosomal thiol reductase; AEP, asparaginyl endopeptidase; CTSB, cathepsin B; TCR, T cell receptor; CD4, T-cell surface glycoprotein. Significance was evaluated by one-way ANOVA followed by Tukey multiple range tests. FM, control diet; bL, containing $1.5 \% 7 \mathrm{~S}$ diet, bH, containing $6 \%$ 7S diet, bH-NaB, containing $6 \% 7 \mathrm{~S}$ and $0.13 \% \mathrm{NaB}$ diet. a, b, c Mean values denoted by different superscripted letters differ significantly at the $P<0.05$ level $(P<0.05)$.

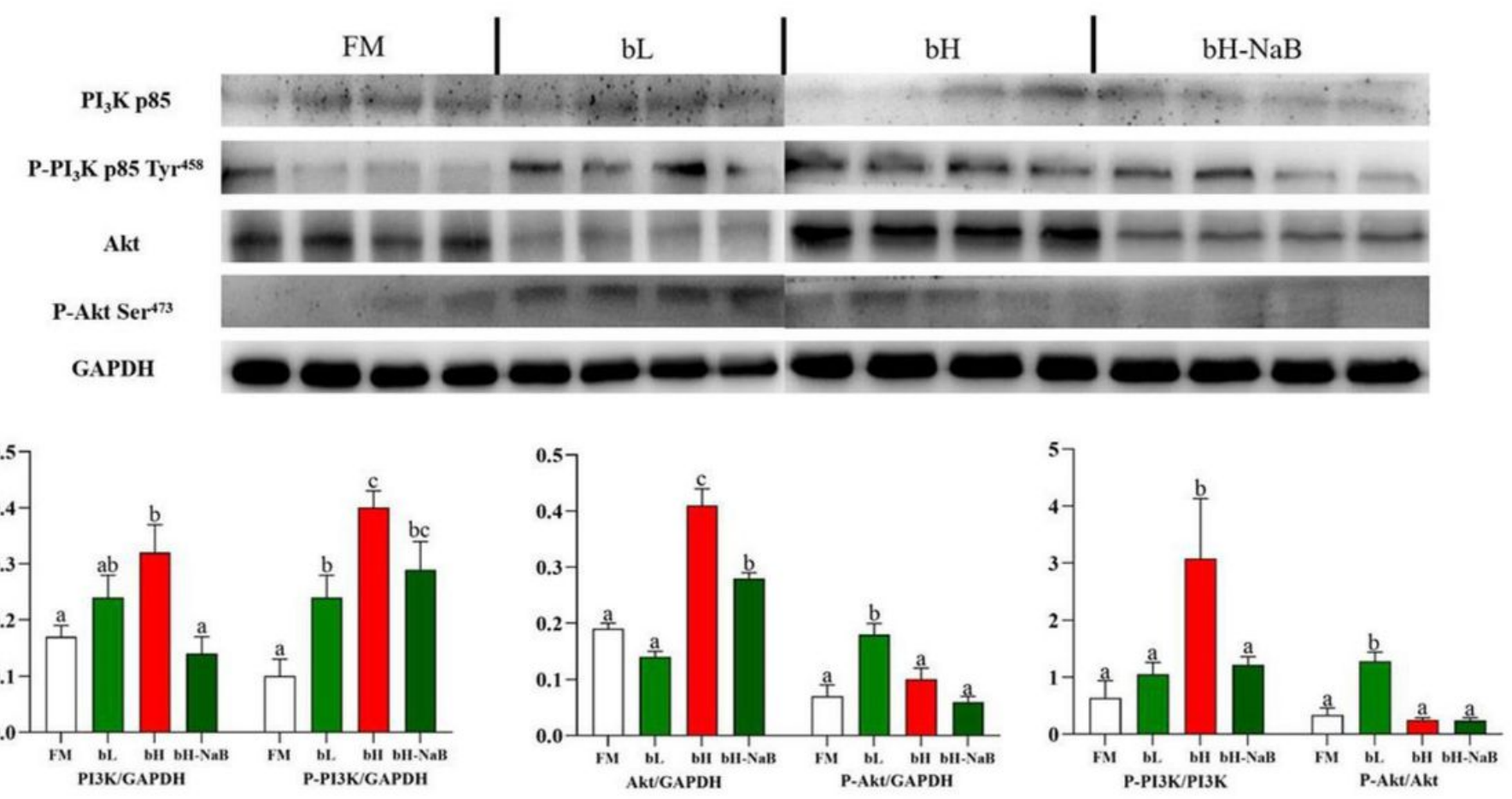

Figure 6

Dietary modulations of distal intestinal responses in the target of PI3K/Akt/mTOR signaling pathway. The samples derive from the same experiment and that gels and blots were processed in parallel. Results are represented as mean $\pm S E(n=4)$. Significance was evaluated by one-way ANOVA followed by Tukey's multiple range tests. $\mathrm{FM}$, control diet; bL, containing $1.5 \% 7 \mathrm{~S}$ diet, $\mathrm{bH}$, containing $6 \% 7 \mathrm{~S}$ diet, bH-NaB, containing $6 \% 7 \mathrm{~S}$ and $0.13 \% \mathrm{NaB}$ diet. $a, b, c$ Mean values among all treatments with different letters were significantly different when the interaction was significant $(P<0.05)$.
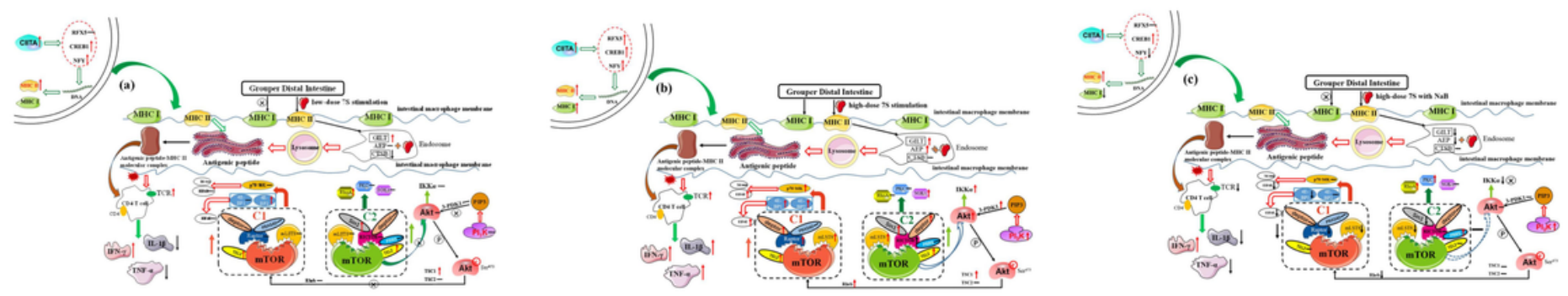

Figure 7 
Summary of the potential pathways associated with exogenous antigen $\beta$-conglycinin (7S) processing, presentation, and CIITA-mediated MHC II-PI3K/Akt/mTOR in the distal intestine of juvenile hybrid groupers (Epinephelus fuscoguttatus $₫ \times \mathrm{E}$. lanceolatus $₫$ ). (a): the bL group (diet containing $1.5 \% 7 \mathrm{~S}$ ); (b): the bH group (diet containing $6 \% 7 \mathrm{~S}$ ); (c): the bH-NaB group (diet containing $6 \% 7 \mathrm{~S}$ and $0.13 \% \mathrm{NaB}$ ).

\section{Supplementary Files}

This is a list of supplementary files associated with this preprint. Click to download.

- SupplementaryMaterial.pdf 